\title{
Elaboración y caracterización de un producto preformado congelado "listo para el consumo" a base de músculo de bonito (Sarda chiliensis)
}

\author{
Elaboration and characterization of a frozen preformed product" ready for consumption "based on \\ muscle of bonito (Sarda chiliensis)
}

\author{
Juan Rodolfo Omote Sibina ${ }^{1 *}$; Andrés Molleda Ordoñez ${ }^{2}$ \\ * Autor de correspondencia
}

\section{Resumen}

Se elaboró un producto preformado congelado "listo para el consumo" a partir de músculo desmenuzado de bonito (Sarda chiliensis) con inclusión de quinua (Quenopodium quinoa willd), con la finalidad de darle mayor valor agregado al recurso y asi promover su consumo. Para ello se elaboraron nueve formulaciones y una muestra control solo con pescado; las variables de estudio fueron polifosfato $(0,1 \%, 0,3 \%$ y $0,5 \%)$, quinua $(3 \%, 6 \%$ y $9 \%)$, mantequilla y aceite $(2 \%, 4 \%$ y $6 \%)$ respectivamente. Para la evaluación sensorial se analizaron las muestras a través de un panel de 15 jueces semi entrenados y para la determinación de los parámetros de textura se utilizó un texturómetro, BrookfieldFarnell QTS - 25. Los resultados de los estudios indicaron que el polifosfato al 0,5\%, quinua al $6 \%$ y mantequilla $4 \%$ mejoraron la textura en función de la jugosidad y sabor del producto, respectivamente. Asimismo, se obtuvo que la forma final aceptada del preformado congelado de músculo desmenuzado de bonito fue cuadrada con un espesor de 1,5 cm, la cual tuvo un tiempo de congelado de tres horas con 20 minutos. Por tanto, a partir de estos resultados la formulación final fue: pulpa molida de bonito (78 \%), zanahoria (4\%), cebolla (6\%), quinua (6\%), sal (1\%), pimienta $(0,1 \%)$, polifosfato $(0,5 \%)$, Glutamatomonosódico (0,3\%) y mantequilla (4\%).

Palabras clave: formulación; caracterización; preformado; bonito; quinua; polifosfato; glutamato monosódico; texturómetro; evaluación sensorial.

\begin{abstract}
A preformed frozen product was prepared "ready for consumption" from shredded bonito muscle (Sarda chiliensis) including quinoa (Quenopodium quinoa willd), in order to give greater added value to the resource and thus promote its consumption. For this, nine formulations and a control sample with only fish were prepared; the study variables were polyphosphate $(0,1 \%, 0,3 \%$ and $0,5 \%)$, quinoa (3\%, $6 \%$ and $9 \%)$, butter and oil $(2 \%, 4 \%$ and $6 \%)$ respectively. For the sensory evaluation the samples were analyzed through a panel of 15 semi-trained judges and for the determination of the texture parameters a texturometer, Brookfield-Farnell QTS-25 was used. The results of the studies indicated that the polyphosphate at $0,5 \%$, quinoa at $6 \%$ and butter $4 \%$ improved the texture depending on the juiciness and flavor of the product, respectively. Likewise, it was obtained that the final accepted form of the preformed frozen crushed muscle of bonito was square with a thickness of $1,5 \mathrm{~cm}$, which had a freezing time of three hours with 20 minutes. Therefore, from these results the final formulation was: ground pulp of bonito (78 \%), carrot (4\%), onion (6\%), quinoa (6\%), salt (1\%), pepper $(0,1 \%))$, polyphosphate $(0,5 \%)$, glutamate-sodium $(0,3 \%)$ and butter $(4 \%)$.
\end{abstract}

Keywords: Formulation; characterization; preformed; bonito; quinoa; polyphosphate; monosodium glutamate; texturometer; sensory evaluation.

\section{Introducción}

El sector pesquero en el Perú tiene una tradicional importancia que está sustentada en los recursos pesqueros marinos pelágicos como la anchoveta, sardina, bonito, jurel y caballa que se encuentran en las aguas jurisdiccionales del Perú (FAO, 2010).

El bonito es una especie cuyo desembarque en el Perú bordea aproximadamente las 116,995 mil toneladas para el año 2016 - mayo 2017, y su consumo está destinado principalmente al estado fresco $(90 \%)$ y enlatado $(4,5$ \%) (PRODUCE, 2017); constituye uno de los principales recursos pesqueros de alto consumo y preferencia de los peruanos (PRODUCE, 2015). Esta especie posee en su composición todos los aminoácidos esenciales, además presenta un alto contenido de ácidos grasos poliinsaturados como DHA y EPA, así como minerales y vitaminas, lo que

\footnotetext{
${ }^{1}$ Universidad Nacional Agraria La Molina, Lima 12, La Molina, Lima-Perú. Email: romote@lamolina.edu.pe

${ }^{2}$ Universidad Nacional Agraria La Molina, Lima 12, La Molina, Lima, Perú. Email: amolleda@lamolina.edu.pe
} 
lo convierte en un alimento de alta calidad nutricional (Dávalos, 2016). Por otro lado, la quinua posee altos niveles de almidón (58\%), que podrían otorgar un poder aglutinante al producto final. Además, este pseudo cereal se caracteriza por tener un alto nivel proteico $(13,8 \%$ $21,9 \%$ ), que también podrían mejorar el nivel proteico contenido en la formulación final. (Llorente, 2008). Las personas actualmente debido a su ritmo de vida, el tiempo que tiene es cada vez más escaso por lo que el tiempo que se demoran en recibir, preparar y consumir son una de las razones más importantes en la decisión a la hora de comprar, además de que prima la calidad tanto en la elaboración como en el aporte nutricional (Pérez,2016). Razones por las cuales se elaboró y caracterizó un producto preformado congelado "listo para el consumo" a base de músculo de bonito (Sarda chiliensis) y quinua (Chenopodium quinoa willd), los objetivos fueron: determinar el porcentaje de inclusión de quinua, seleccionar el tipo de materia grasa, determinar el porcentaje de polifosfato en la mezcla, determinar la forma y espesor del producto final, evaluar a calidad del producto final, mediante análisis sensorial, químico y microbiológico.

\section{Materiales y métodos}

El trabajo de investigación se llevó a cabo en los laboratorios de Microbiología Pesquera, Química de Recursos Hidrobiológicos y Procesos de la Facultad de Pesquería de la Universidad Nacional Agraria La Molina.

\section{Materia prima e insumos}

Se utilizó como materia prima la especie Sarda chiliensis (Bonito), proveniente del terminal Pesquero de Villa María. Fue adquirida al estado fresco y entera, las unidades fueron colocadas en cajas isotérmicas limpias y cubiertas con hielo en una proporción de 2:1 (hielo:pescado) para su traslado al laboratorio de procesos de la Facultad de Pesquería de la UNALM. Por otro lado, se utilizó quinua, proveniente del mercado de productores. Otros insumos que ingresaron en la elaboración fueron Mantequilla con sal, aceite vegetal (girasol), zanahoria, cebolla blanca, sal (cloruro de sodio), pimienta negra, polifosfato de sodio, glutamato monosódico.

\section{Análisis Físico - Sensorial}

Se registró la longitud total con el ictiómetro, con el fin de verificar que el tamaño se encuentre dentro del rango de talla mínima indicada por PRODUCE (2001), y el peso para evaluar los rendimientos en cada etapa del proceso (Ostalaza, 1978). Así mismo, se evaluó el estado de frescura de manera sensorial utilizando la Tabla de Wittfogel, propuesta por Teruya (1973).

\section{Análisis Químico Proximal}

La composición química proximal de la materia prima y del producto final se realizó según el procedimiento descrito por la AOAC (2005), realizándose las siguientes pruebas: - Humedad, (método 925,10), - Cenizas, (método 942,05), - Grasa por método Soxhlet con hexano (método 203,05), Proteínas por método Kjeldahl. (método 984,13).

\section{Análisis Sensorial}

Para el análisis sensorial del producto, se utilizó la metodología propuesta por Stejkal et al. (2011). Se seleccionó un panel conformado por 15 personas, quienes fueron capacitados a través de una presentación donde se enfatizó las características que se quería evaluar. Las muestras a evaluar fueron preparadas a través de una cocción por microondas.

\section{Determinación del pH}

Se utilizó el método descrito por Pearson (1993).

\section{Análisis de Perfil de Textura (TPA)}

Se utilizó el texturómetro, Brokfield - Farnell QTS - 25, y se realizó según la metodología propuesta por Shaviklo et al. (2013). Las condiciones fueron: - Dimensión de la muestra: $30 \mathrm{~mm}$ x $30 \mathrm{~mm} \times 10 \mathrm{~mm}$.

\section{Análisis Microbiológico}

Los análisis se realizaron en el producto final, siguiendo lo propuesto por Ingram et al. (1983). Las pruebas fueron: - Numeración de aerobios mesófilos. - Numeración de coliformes totales y coliformes fecales. - Numeración de Staphylococcus aureus. - Detección de Salmonella sp.

\section{$\underline{\text { Curva de Congelado }}$}

Para la elaboración de esta curva, se utilizó un congelador horizontal doméstico con una temperatura interna máxima de $-30{ }^{\circ} \mathrm{C}$ y se monitoreo la temperatura interna a través de un termómetro digital con una termocupla y se realizó según la metodología propuesta por Muela et al. (2011).

\section{Análisis estadístico}

Los resultados de las pruebas de análisis sensorial efectuadas y análisis de perfil de textura (TPA) fueron analizadas por medio del ANOVA, y la prueba comparativa de Dunett a un nivel de significancia de 0,05 , para ello se utilizó el programa estadístico MINITAB 2016. La prueba de aceptabilidad del producto final fueron analizados según el método sensorial denominado Ordenamiento para Análisis Afectivos, analizados por el método estadístico de Friedman (Conover, 1980), con un nivel de significancia del 0.05 .

\section{Procedimiento}

Para la elaboración de la hamburguesa la materia prima fue trasladada en cajas isotérmicas acondicionadas con hielo en una relación de 2:1 a una temperatura entre 1 5 
${ }^{\circ} \mathrm{C}$, adquirido en el Terminal Pesquero de Villa María. Se evaluó sensorialmente de acuerdo a Tabla de evaluación organoléptica de Wittfogel (Teruya, 1973). El bonito fue descabezado y eviscerado de forma manual aplicando un corte por detrás de la aleta pectoral para eliminar la cabeza y el contenido visceral, luego fue lavado para eliminar restos de vísceras. Una vez limpio, se procedió a filetear eliminando piel y espinas. Los filetes limpios se colocaron en canastillas de plástico y sumergidos en una salmuera de baja concentración $(3 \% \mathrm{p} / \mathrm{v})$ a una temperatura entre $0 \sim 5{ }^{\circ} \mathrm{C}$ por un tiempo de 5 a 10 segundos para poder darle textura y eliminar coágulos de sangre, se escurrieron en una canastilla de plástico por un tiempo de $10 \mathrm{~min}$ y llevados a una moledora de carne con una criba de $5 \mathrm{~mm}$. El filete molido se colocó en una mezcladora con los demás insumos por un tiempo de cinco minutos a una temperatura constante de $5{ }^{\circ} \mathrm{C}$ hasta obtener una masa homogénea. Luego se preformó de forma manual para darle la forma y espesor. Se procedió a congelar los preformados de bonito en un congelador horizontal doméstico, midiendo la temperatura interna cada 5 minutos hasta llegar a $-18^{\circ} \mathrm{C}$. Una vez congelado se colocaron en bolsas de polietileno de alta densidad y se almacenó a una temperatura de $-20{ }^{\circ} \mathrm{C}$.

La investigación se realizó en cuatro etapas: la primera en donde se determinó el porcentaje de polifostafo $(0,1$; 0,$3 ;$ y $0,5 \%$ ), la segunda etapa contempló la determinación de la cantidad de quinua en la formulación (3, 6 y $9 \%$ ), en la tercera etapa se determinó entre mantequilla y aceite cuál de ellos presentaba mejor sabor en el producto preformado para ello se probaron porcentajes de 2, 4 y $6 \%$ respectivamente, en la última etapa se estudió cual era la mejor forma (circular, cuadrada, romboide) y espesor (1; 1,5 y $2 \mathrm{~cm}$ ). Los datos obtenidos de cada experimento fueron analizadas por medio de un ANOVA con una significancia de $0,05 \%$, y a través de la prueba no paramétrica de Friedman para medir la aceptabilidad del producto preformado.

\section{Resultados y discusión}

\section{Materia Prima}

Análisis físico - sensorial

Los resultados obtenidos al realizar la evaluación sensorial a las unidades de bonito, según la Tabla de Wittfogel (Teruya, 1973) se muestran en la Tabla 1, los resultados obtenidos determinaron que la materia prima utilizada era de calidad comercial EXTRA.

Se determinó también el peso y longitud promedio de las unidades de bonito las cuales tuvieron como promedio $3,10 \mathrm{~kg}$ y $56,5 \mathrm{~cm}$, respectivamente, lo que nos indicó que las muestras se encontraban dentro del rango de talla y peso de un pez maduro, reportado por IMARPE, 2001 (Tabla 2).

\section{Análisis Químico - Proximal de la materia prima}

Se realizó el análisis químico proximal en el músculo de bonito fresco, los resultados obtenidos se presentan en la Tabla 3.
Tabla 1. Resultados del análisis sensorial

\begin{tabular}{lc}
\hline \multicolumn{1}{c}{ Característica } & Puntaje \\
\hline Superficie y consistencia & 4 \\
Ojos & 4 \\
Branquias & 3 \\
Cavidad abdominal y órganos & 4 \\
Olor & 3 \\
\hline Total & $18 / 20$ \\
\hline
\end{tabular}

Tabla 2. Resultados talla y peso del bonito

\begin{tabular}{lcc}
\hline & Resultados & IMARPE 2001 \\
\hline Talla $(\mathrm{cm})$ & 56,5 & 52 \\
Peso $(\mathrm{kg})$ & 3,10 & 2,3 \\
\hline
\end{tabular}

Tabla 3. Composición química proximal del músculo de bonito

\begin{tabular}{ccccc}
\hline Componente & $\begin{array}{c}\text { Muestra } \\
\%\end{array}$ & $\begin{array}{c}\text { Ostalaza } \\
(1987) \\
\%\end{array}$ & $\begin{array}{c}\text { Teruya } \\
(1973) \\
\%\end{array}$ & $\begin{array}{c}\text { IMARPE } \\
(1996) \%\end{array}$ \\
\hline Humedad & 73,30 & 75,80 & 72,14 & 70,80 \\
Ceniza & 1,08 & 1,38 & 1,32 & 1,20 \\
$\begin{array}{c}\text { Proteína }(\mathrm{N} \mathrm{x} \\
\text { 6,25) }\end{array}$ & 20,55 & 21,55 & 19,96 & 19,10 \\
Grasa & 5,07 & 1,27 & 5,09 & 8,20 \\
\hline
\end{tabular}

Los valores de ceniza y proteína obtenidos en el trabajo de investigación son similares a los reportados por Ostalaza (1978), Teruya (1973) y el IMARPE (1996). Sin embargo, se observó diferencias en el contenido de humedad y grasa, variando 4 y $3 \%$, respectivamente. Al respecto, Ostalaza (1978), menciona que el contenido de proteína en el bonito puede bajar debido a épocas cercanas de ovulación. Por otro lado, Sirkoski (1994), mencionó una relación inversa entre el contenido de humedad y grasa con la madurez sexual, a mayor humedad menor grasa. Por lo que es probable que la especie se encontraba en plena maduración.

\section{Determinación del porcentaje polifosfato}

El panel de 15 degustadores, evaluaron los atributos establecidos para la textura como: elasticidad, firmeza, dureza y suculencia. Los resultados obtenidos del análisis sensorial por atributos fueron por escala en donde el rango de la elasticidad y firmeza se evaluaron de 1 a 5 (mala a buena), dureza en un rango de 1-5 (duro a suave) y la suculencia en un rango de 1-5 (seco a jugoso) se detallan en la Tabla 4.

Las variables de los atributos dureza y suculencia fueron los que presentaron diferencias significativas $(\mathrm{p}<0,05)$, mientras que para los atributos de elasticidad y firmeza no se observaron diferencias por tanto no tuvieron mayor relevancia para la elección de la mejor variable. De acuerdo al análisis de variancia realizado la muestra con mayor preferencia fue la del tratamiento A3 (0,5\% de polifosfato), la cual obtuvo una puntuación de 4,1 y 4,2 en los atributos de dureza y suculencia, respectivamente. 
Estos resultados son similares a los reportados por Gonçalves y Duarte (2009), quienes comprobaron que el polifosfato incrementa la capacidad de retención de agua en el langostino rojo, por tanto aumenta la aceptación en los consumidores.

Tabla 4. Promedio de calificaciones obtenidas sensorialmente para el polifosfato A1, A2 y A3

\begin{tabular}{lccccc}
\hline Atributo & & C & A1 & A2 & A3 \\
\hline Elasticidad & n.s. & 3,4 & 3,5 & 3,8 & 3,5 \\
Firmeza & n.s. & 3,5 & 3,6 & 3,7 & 3,7 \\
Dureza & s & $3,2 \mathrm{a}$ & 3,5 & 3,3 & $4,1 \mathrm{a}$ \\
Suculencia & s & $3,2 \mathrm{a}$ & 3,4 & 3,8 & $4,2 \mathrm{a}$ \\
\hline
\end{tabular}

n.s: no significativo, s: significativo

Panuncio et al. (2013), mencionan que dentro de una prueba sensorial la dureza representa un atributo importante para el paladar del consumidor, debido a que al utilizar una menor fuerza para comprimir la muestra entre los dientes facilitará su trituración. De igual importancia es la suculencia, ya que por medio de esta se perciben los jugos de la carne afectando la palatabilidad, además ayudan al ablandamiento y a la fragmentación de la carne durante la masticación (Geat et al., 2001).

En la Figura 1 se observa como la adición del polifosfato afectó sensiblemente los valores de $\mathrm{pH}$ respecto a la muestra control $(5,76)$, observándose un aumento gradual de acuerdo al porcentaje de adición del polifosfato en el músculo desmenuzado preformado

de bonito. Pessanha (2004), menciona que conforme aumenta la adición de polifosfato en la carne, se observará un aumento gradual de $\mathrm{pH}$, confirmándose lo obtenido

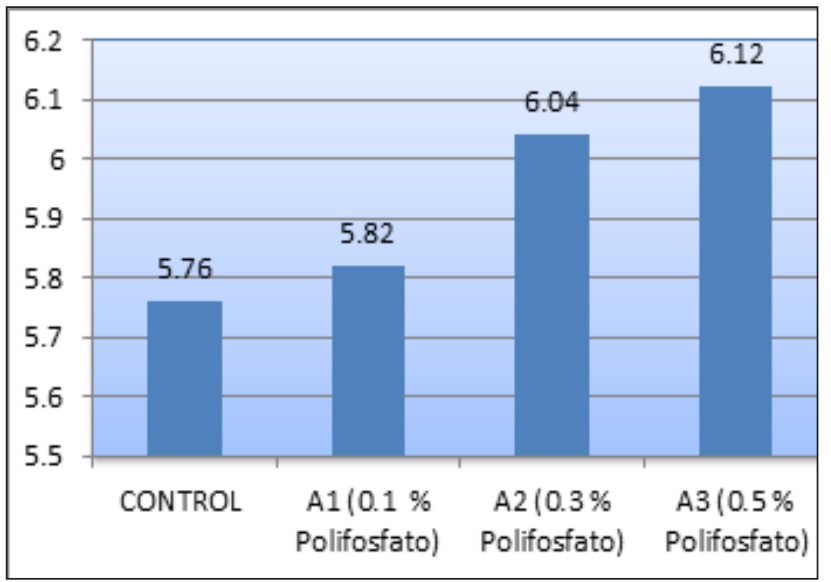

Figura 1. Valores de $\mathrm{pH}$ de las tabletas de bonito de la muestra control y de los variables A1 $(0,1 \%), \mathrm{A} 2(0,3 \%)$, y A3 $(0,5 \%)$

\section{Determinación del porcentaje de quinua}

Se evaluó sensorial e instrumentalmente tres muestras de músculo desmenuzado preformado de bonito con diferentes porcentajes de quinua: B1 (3\%), B2 (6\%) y B3 (9\%), y se comparó con la muestra control. Los resultados obtenidos del análisis sensorial por atributos fueron por escala en donde el rango de la elasticidad y firmeza se evaluaron de 1 a 5 (mala a buena), dureza en un rango de 1-5 (duro a suave) y la suculencia en un rango de 1-5 (seco a jugoso) se detallan en la Tabla 5.

Tabla 5. Promedio de puntajes obtenido de los atributos evaluados de B1, B2 Y B3

\begin{tabular}{cccccc}
\hline ATRIBUTO & & $\mathrm{C}$ & $\mathrm{B} 1$ & $\mathrm{~B} 2$ & $\mathrm{~B} 3$ \\
\hline Elasticidad & n.s & 3,4 & 3,18 & 4,2 & 3,27 \\
Firmeza & n.s & 3,5 & 3,33 & 3,73 & 3,73 \\
Dureza & $\mathrm{s}$ & $3,2 \mathrm{ab}$ & $3,87 \mathrm{a}$ & $4,0 \mathrm{~b}$ & 3,42 \\
Suculencia & $\mathrm{s}$ & $3,2 \mathrm{abc}$ & $3,79 \mathrm{a}$ & $3,98 \mathrm{~b}$ & $2,86 \mathrm{c}$ \\
\hline
\end{tabular}

n.s: no significativo, s: significativo. valores con diferente letra denota diferencias entre los tratamientos en función del control.

Los atributos de dureza y suculencia presentaron diferencias significativas $(\mathrm{p}<0,05)$. El análisis estadístico del atributo dureza, en cuanto a las muestras se determinó que existieron entre la muestra control con las variable B1 (3\% de quinua) y B2 (6\% de quinua), observando la mayor diferencia entre la muestra control y la variable B2 $(6 \%)$.

Shaviklo et al. (2013), mencionan que los atributos más importante dentro del proceso de degustación son la dureza y la suculencia. Además, Dransfield et al. (1984), mencionan que la terneza (contrario a la dureza) y la jugosidad explican una importancia de $64 \%$ y $19 \%$, respectivamente, en las diferencias entre las muestras. En ambos atributos (dureza y suculencia), el tratamiento B2 (6 $\%$ de quinua) fue la de mayor preferencia, ya que tenía una textura agradable y presento la jugosidad necesaria. Además se debe mencionar que la jugosidad está relacionada con la suculencia, ya que esta última se define como la cantidad y modo que el jugo es expulsado y la forma que se percibe la carne tras su deglución, registrada durante la constante masticación de todo el bolo y mediante la cantidad de jugo que va siendo expulsada (Harries et al., 1972).

De otro lado los resultados al analizar el perfil de textura (Tabla 6) por medio del texturómetro evidenció que existen diferencias significativas entre los atributos de dureza, elasticidad y masticabilidad, esto puede ser afectado por la presencia de agentes espesantes, aglutinantes y formadoras de gel en la quinua como es el almidón (Hevia et al., 2000).

Determinación del contenido de mantequilla y/o aceite en la mezcla

Las pruebas de preferencia, según los atributos de sabor, olor textura, y el análisis de comparativo de Dunnet realizada en las muestras de músculo desmenuzado preformado de bonito con mantequilla y aceite, indicaron que hubo diferencias significativas $(p<0,05)$ en los atributos de sabor y textura (Tabla 7 y 8 ). Se calificaron a través de una Tabla con un rango de $1-5$ (muy malo - muy bueno). Se 
observó que en estos dos atributos hubo diferencias entre la muestra control y la variable C2 (4\% de mantequilla). Respecto al sabor, los panelistas percibieron un sabor más agradable, mientras que para la textura indicaron que fue un producto suave y jugoso para el paladar. Respecto a las muestras con aceite evaluadas el atributo sabor, se observó que hubo diferencia significativa entre la muestra control y la variable D3 (6 \% de aceite), debido a que esta última presentó un sabor poco paladeable, además los panelistas indicaron que percibieron un exceso de aceite en el producto. Las muestras obtenidas de las variables D1 (2\% de aceite) y D2 (4\% de aceite), no presentaron diferencias significativas respecto a la muestra control, los panelistas afirmaron que presentaba un sabor agradable. Con respecto a la textura los panelistas indicaron que la variable D2 (4 $\%$ ) presentó una textura más consistente pero a la vez suave al paladar, mientras que la muestra D3 (6\%) presentó una textura más blanda, teniendo una consistencia suave. Por tal motivo, podemos decir que fue la variable D2 (4\%) la de mayor preferencia.

Los atributos de sabor, intensidad de sabor, humedad bucal y textura, son los que influencian en mayor medida en la determinación de análisis sensorial (Panuncio et al. 2013). Tokur et al. (2005), también consideró el sabor como el principal atributo el cual afectará la aceptabilidad del producto. Además Calvo et al. (2007), mencionan que el sabor es el principal beneficio percibido por los consumidores en el momento de ingerirlo. En cuanto al análisis del perfil de textura (TPA) realizado a las muestras que contenían mantequilla y aceite (Tabla 9 y 10), se determinó que en las muestras preformadas congeladas de bonito con mantequilla la dureza aumentó en un 51,21 $\%$ en relación con la muestra control, se observó una relación directamente proporcional entre la masticabilidad y la dureza para las variables C1 (2\% de mantequilla) y C3 (6\% de mantequilla) mientras que para la muestra C2 (4\% de mantequilla) presentó una disminución de la masticabilidad. Hernández (2005) menciona que la elasticidad será un atributo relevante solo para hallar la masticabilidad. La muestra evaluada con aceite se observó que la dureza de la variable D2 (4\%) aumentó en un $46,74 \%$ en relación a la muestra control. Sin embargo, la variable D3 disminuyó a 33,35 \% y con ello disminuyo la masticabilidad.

Tabla 6. Caracterización de la textura de las muestras por método de Análisis de Perfil de Textura (TPA)

\begin{tabular}{|c|c|c|c|c|c|}
\hline ATRIBUTO & & $\mathrm{C}$ & B1 & $\mathrm{B} 2$ & B3 \\
\hline Dureza (N) & $\mathrm{s}$ & $25,98 \pm 1,96 a b c$ & $45,98 \pm 1,26 a$ & $49,01 \pm 1,623 b$ & $62,67 \pm 1,247 \mathrm{c}$ \\
\hline Cohesividad & n.s. & $0,55 \pm 0,045$ & $0,54 \pm 0,035$ & $0,505 \pm 0,023$ & $0,54 \pm 0,018$ \\
\hline Elasticidad & $\mathrm{s}$ & $0,45 \pm 0,07 \mathrm{ab}$ & $0,53 \pm 0,022 \mathrm{a}$ & $0,5 \pm 0,01$ & $0,62 \pm 0,013 b$ \\
\hline Masticabilidad (N) & $\mathrm{s}$ & $6,43 \pm 0,546 \mathrm{abc}$ & $13,16 \pm 0,646 a$ & $12,37 \pm 048 b$ & $1,98 \pm 0,921 \mathrm{c}$ \\
\hline
\end{tabular}

s: signifcancia, n.s. no significancia, valores con diferente letra denota diferencias entre los tratamientos en función del control.

Tabla 7. Calificaciones de los atributos del producto con mantequilla al 2, 4 y $6 \%$

\begin{tabular}{cccccc}
\hline Atributos & & Control & C1 & C2 & C3 \\
\hline Sabor & s & $3,1 \mathrm{a}$ & 2,9 & $4,4 \mathrm{a}$ & 2,5 \\
Olor & N.S & 2,9 & 3,1 & 3,0 & 2,9 \\
Textura & s & $3,0 \mathrm{a}$ & 3,1 & $3,9 \mathrm{a}$ & 3,0 \\
\hline n.s: no significativo, s: significativo & & &
\end{tabular}

Tabla 9. Caracterización de la textura de las muestras con mantequilla por método de TPA

\begin{tabular}{lccccc}
\hline ATRIBUTO & & CONTROL & C1 & C2 & C3 \\
\hline Dureza (N) & $\mathrm{s}$ & $25,98 \pm 1,96 \mathrm{abc}$ & $49,001 \pm 1,37 \mathrm{a}$ & $50,34 \pm 1,775 \mathrm{~b}$ & $53,25 \pm 1,98 \mathrm{c}$ \\
Cohesividad & $\mathrm{n} . \mathrm{s}$ & $0,55 \pm 0,045$ & $0,53 \pm 0,043$ & $0,49 \pm 0,0275$ & $0,54 \pm 0,021$ \\
Elasticidad & $\mathrm{s}$ & $0,45 \pm 0,07 \mathrm{a}$ & $0,575 \pm 0,031 \mathrm{a}$ & $0,5175 \pm 0.02$ & $0,517 \pm 0,022$ \\
Masticabilidad (N) & $\mathrm{s}$ & $6,43 \pm 0,54 \mathrm{abc}$ & $13,99 \pm 1,37 \mathrm{a}$ & $12,88 \pm 0,754 \mathrm{~b}$ & $15,027 \pm 1,21 \mathrm{c}$ \\
\hline
\end{tabular}

s: signifcancia, n.s. no significancia, valores con diferente letra denota diferencias entre los tratamientos en función del control

Tabla 10. Caracterización de la textura de las muestras con aceite por método de TPA

\begin{tabular}{cccccc}
\hline ATRIBUTO & & CONTROL & D1 & D2 & D3 \\
\hline Dureza (N) & s & $25,98 \pm 1,96 \mathrm{abc}$ & $45,97 \pm 2,253 \mathrm{a}$ & $48,84 \pm 1,772 \mathrm{~b}$ & $38,98 \pm 1,571 \mathrm{c}$ \\
Cohesividad & N.S. & $0,55 \pm 0,045$ & $0,64 \pm 0,006$ & $0,63 \pm 0,021$ & $0,64 \pm 0,034$ \\
Elasticidad & $\mathrm{s}$ & $0,45 \pm 0,07 \mathrm{ab}$ & $0,31 \pm 0,056 \mathrm{a}$ & $0,43 \pm 0,017$ & $0,33 \pm 0,016 \mathrm{~b}$ \\
Masticabilidad (N) & $\mathrm{s}$ & $6,43 \pm 0,54 \mathrm{abc}$ & $13,23 \pm 1,46 \mathrm{a}$ & $13,19 \pm 0,36 \mathrm{~b}$ & $8,18 \pm 0,307 \mathrm{c}$ \\
\hline
\end{tabular}

s: signifcancia, n.s. no significancia, valores con diferente letra denota diferencias entre los tratamientos en función del control 
Se determinó que las mejores variables tanto para la mantequilla como para el aceite fueron el C2 (4\% de mantequilla) y el D2 (4\% de aceite) respectivamente. Los resultados de la Tabla 11 indican que hubo diferencias significativas $(p<0,005)$ entre las muestras.

Tabla 11. Calificaciones de los atributos del producto $\mathrm{C} 2$ y D2

\begin{tabular}{llccc}
\hline \multicolumn{2}{l}{ ATRIBUTOS } & CONTROL & C2 & D2 \\
\hline Sabor & S & $3,1 \mathrm{a}$ & $4,4 \mathrm{a}$ & 3,2 \\
Olor & N.S & 2,9 & 3,1 & 3,0 \\
Textura & s & $3,0 \mathrm{ab}$ & $3,8 \mathrm{a}$ & $3,5 \mathrm{~b}$ \\
\hline
\end{tabular}

n.s: no significativo, s: significativo

Las mejores características la presentó el producto obtenido con el tratamiento $\mathrm{C} 2$, los panelistas manifestaron un sabor agradable, mientras que al probar el tratamiento D2 percibieron una sensación "grasosa" en el paladar, siendo rechazada por los mismos. Según Wright et al. (2001), indican que la textura que proporciona la mantequilla como un insumo, es considerada un atributo importante en la elección del consumidor, esto debido a que las propiedades de la mantequilla están relacionadas íntimamente a su estructura subyacente.

\section{Determinación de la forma y espesor}

Los resultados se muestran en la Figura 2, se puedo determinar que no existen diferencias significativas entre las formas propuestas, pero dentro de las que obtuvo mayor puntuación se encontró que fue la forma cuadrada con un promedio de 2,32. Muñoz (2011) menciona que la calidad sensorial (la forma, el color, el tamaño), junto con la textura, el sabor y olor, es fundamental para atraer al consumidor, ya que a través de estas, tendrá una experiencia grata con el producto y estará dispuesto a pagar por ellas.

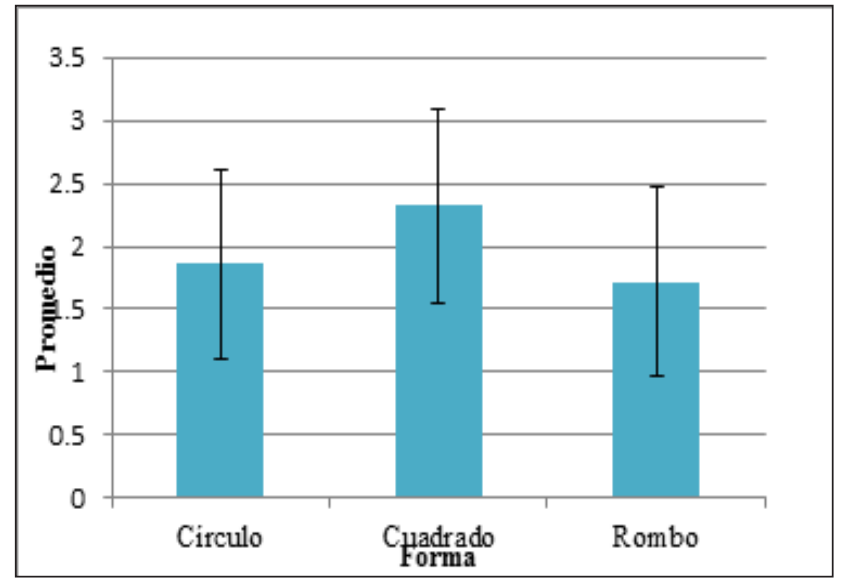

Figura 2. Forma final de las tabletas de pescado

Para determinar el espesor adecuado, se realizó en la muestra de forma cuadrada y por medio de la curva de congelado, la Figura 3, en ella se muestra como variaron los 3 espesores respecto al tiempo de congelación.

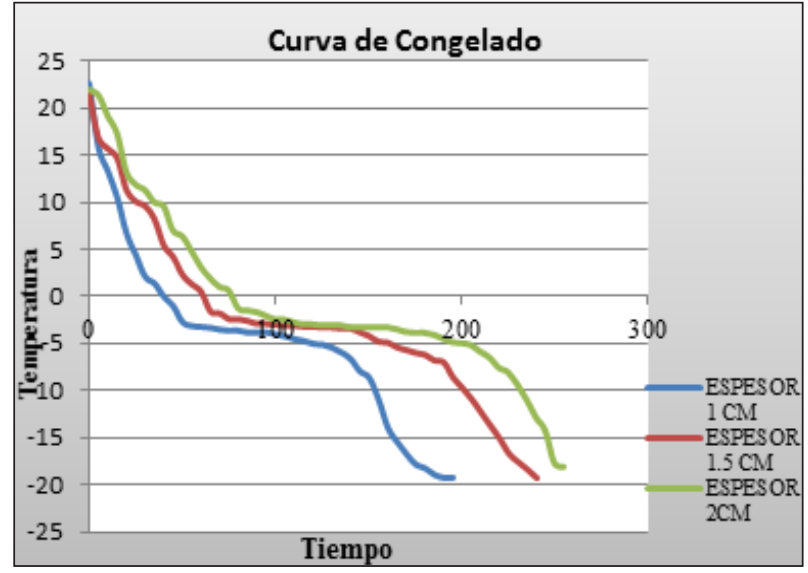

Figura 3. Curva de congelado para diferentes espesores

Se determinó que el espesor adecuado fue el de $1,5 \mathrm{~cm}$ con un tiempo de 225 minutos, debido a que durante el proceso de preparación la de menor espesor presentó una textura más endeble, muy fácilmente fracturable; por otro lado el de mayor espesor si bien no presentó problemas de fracturabilidad si presentó complicaciones durante la preparación para su consumo por el tiempo de cocción, al respecto Farroni (2011) menciona que el espesor es muy importante para la calidad del producto porque si es muy grueso puede resultar de textura dura y si es muy delgada puede romperse demasiado fácil durante el empaque y el transporte.

\section{Caracterización del Producto final}

La formulación final para la elaboración del producto preformado fue: bonito $78,1 \%$, zanahoria $4 \%$, cebolla 6 $\%$, quinua $6 \%$, sal $1 \%$, pimienta $0,1 \%$, polifosfato $0,5 \%$, GMS 0,3\%, mantequilla $4 \%$.

\section{Análisis Químico Proximal}

Los resultados del análisis químico proximal del producto final, se muestran en la Tabla 12.

Tabla 12. Composición química proximal del producto final

\begin{tabular}{lccc}
\hline \multicolumn{1}{c}{ Componente } & Resultados \% & $\begin{array}{c}\text { Martí et al. } \\
(2015) \\
(\mathrm{g} / 100 \mathrm{~g})\end{array}$ & $\begin{array}{c}\text { Melgarejo } \\
(2002) \\
(\mathrm{g} / 100 \mathrm{~g})\end{array}$ \\
\hline Humedad & 66,01 & 73,91 & 72,30 \\
Ceniza & 2,32 & 2,21 & 2,40 \\
Proteína & 17,13 & 19,59 & 18,90 \\
Grasa & 9,02 & 0,72 & 4,85 \\
Carbohidratos & 5,52 & - & 6,52 \\
\hline
\end{tabular}

Respecto al contenido de grasa, dado a la inclusión de la mantequilla como un insumo al igual que la quinua, el producto final presento $9,02 \%$, por encima que los reportados por Melgarejo et al. (2002) y Martí et al. (2015). Mientras que el contenido de ceniza es similar a 
los propuestos por autores mencionados anteriormente.

El contenido de carbohidratos presentó un valor menor al reportado por Melgarejo et al. (2002). Cabe mencionar que, Melgarejo et al. (2002), uso como fuentes carbohidratos galletas y leche en polvo, mientras en el estudio solo se usó quinua cocida.

\section{Análisis Microbiológico}

Los resultados obtenidos (Tabla 13) para los diferentes ensayos realizados se encuentran debajo de los límites establecidos por SANIPES (2016), esto confirma que durante la elaboración del producto se mantuvo la calidad sanitaria, las condiciones de manipuleo y condiciones de higiene de la materia prima.

Tabla 13. Resultados del análisis microbiológico del producto final

\begin{tabular}{lcc}
\hline \multicolumn{1}{c}{ Ensayo } & Resultados & $\begin{array}{c}\text { SANIPES } \\
(2010)\end{array}$ \\
$\begin{array}{l}\text { Numeración de aerobios mesófilos } \\
\text { (UFC/g) }\end{array}$ & $1 \times 1000$ & $10^{6}$ \\
$\begin{array}{l}\text { Numeración de Staphylococcus } \\
\text { aureus (NMP/g) }\end{array}$ & $<3$ & $10^{3}$ \\
$\begin{array}{l}\text { Numeración de coliformes (UFC/g) } \\
\text { Numeración de Escherichia coli } \\
\text { (NMP/g) }\end{array}$ & $1 \times 10$ & $10^{2}$ \\
$\begin{array}{l}\text { Detección de Salmonella sp. (en 25 } \\
\text { g) }\end{array}$ & Ausencia & Ausencia/25 g \\
\hline
\end{tabular}

\section{Análisis de Perfil de textura (TPA)}

Los resultados obtenidos (Tabla 14) respecto a la cohesividad y elasticidad no presentaron mayores diferencias. Mientras en la masticabilidad se observó un valor mucho menor al reportado por Panuncio et al. (2013), quien menciona un valor de $31 \mathrm{~N}$, mientras que el músculo desmenuzado preformado de bonito solo presentó $12.38 \mathrm{~N}$, esto puede ser debido a que la quinua no forma enlaces tan fuertes como la hace la transglutaminasa entre proteínas. La dureza final fue de $49.01 \mathrm{~N}$ superior al reportado por Shaviklo y Fahim (2013), mientras que muestra resultados similares a lo propuesto por Panuncio et al. (2013), donde presenta valores cercanos a la hamburguesa de merluza.

Tabla 14. Análisis de Perfil de textura

\begin{tabular}{cccc}
\hline Atributo & Valor & $\begin{array}{c}\text { Shaviklo y Fahim } \\
(2013)\end{array}$ & $\begin{array}{c}\text { Panuncio } \text { et al. } \\
(2013)\end{array}$ \\
Dureza & $49,01 \mathrm{~N}$ & $20,68 \mathrm{~N}$ & $52,2 \mathrm{~N}$ \\
Cohesividad & 0,51 & 0,62 & 0,67 \\
Elasticidad & 0,50 & 0,46 & 0,89 \\
Masticabilidad & $12,38 \mathrm{~N}$ & $15,31 \mathrm{~N}$ & $31 \mathrm{~N}$ \\
\hline
\end{tabular}

\section{Prueba de aceptabilidad}

Los resultados fueron favorables con relación a sus características sensoriales, puesto que los consumidores indicaron un grado de aceptación y satisfacción alto del producto final. Sin embargo, por las observaciones notadas en las encuestas, nuestra debilidad fue la apariencia del producto.

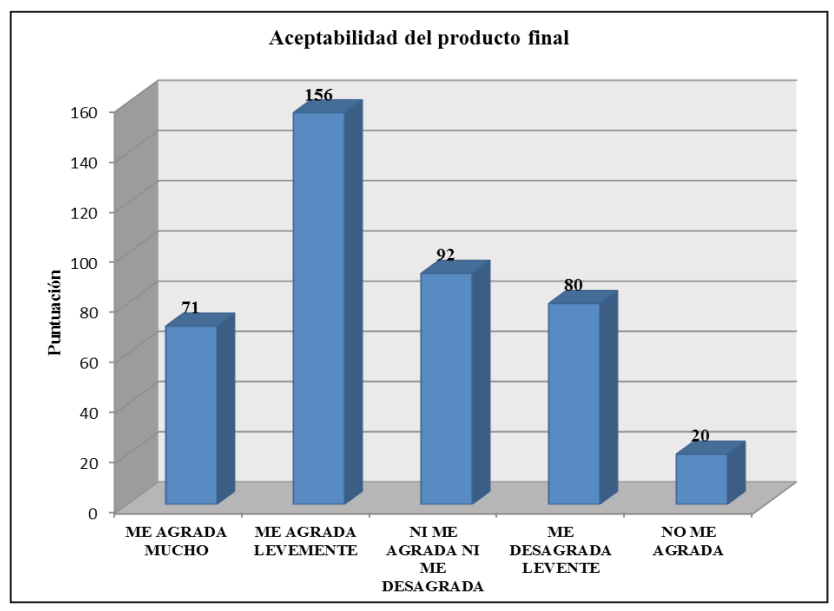

Figura 4. Prueba de preferencia del producto final

\section{Conclusiones}

El flujograma de proceso para la elaboración de músculo desmenuzado preformado de bonito fue: Recepción de materia prima - Descabezado y Eviscerado - Lavado 1 Fileteado - Lavado 2 - Escurrido - Molido - Mezclado - Preformado - Congelado - Envasado - Almacenado. El porcentaje de inclusión del polifosfato fue de 0,5\% (A3). El porcentaje de quinua a incluir en el producto final resulto $6 \%$ (B2). La materia grasa elegida fue la mantequilla al 4 $\%$ (C2). La mejor forma de presentación seleccionada por el panel fue la cuadrada (E2) con un espesor de $1.5 \mathrm{~cm}$ (F2). La formulación final del producto "Preformado de desmenuzado de bonito con quinua" fue: pulpa molida de bonito (78 \%), zanahoria (4\%), cebolla (6\%), quinua (6 $\%)$, sal (1\%), pimienta $(0,1 \%)$, polifosfato $(0,5 \%)$, GMS $(0,3 \%)$ y mantequilla (4\%). El rendimiento final fue de $34,622 \%$ de pulpa de bonito.

\section{Literatura citada}

AOAC [Association of Official Analytical Chemist] .2005. Official Methods Analysis. 18 th ed. AOAC, Washington D.C, 1(3-5):40.

Calvo, D.; Ottar, S. y Tudoran, A. 2007. Análisis de las preferencias para un nuevo producto de pescado de conveniencia: una aplicación empírica para España y Noruega. Grupo de Investigación FEDRA. La Coruña, España. 1 - 13p.

Conover, W. 1980. Practical nonparametric statistics. John Wiley \& Sons, New York. 493p.

Dávalos, C. 2016. Desarrollo de Nuggets de Bonito (Sarda chiliensis chiliensis) bajos en calorías y con la adición de Chia (Salvia hispanica) como Antioxidante (en línea). Tesis Ing. Pesq. Arequipa, Perú, UNSA. 110p. Disponible en: 
http://repositorio.unsa.edu.pe/bitstream/handle/ UNSA/2366/IPlumedc.pdf?sequence $=1$

Dransfield, E.; Francombe,M.A. and Whelehan, O.P. 1984. Relationships between sensory attributes in cooked meat. J. Text. Studies, 15: 37-356.

Farroni, A. 2011. Transformación estructural y físicoquímicas de maíces argentinos en la producción de alimentos obtenidos por procesos de gelatinización - laminación. Tesis doctoral. Universidad de Buenos Aires (en Línea). Disponible en: http://imarca.com.ve/ Formadora-de-Carnes-y-hamburguesas.php

FAO, 2010. Perfíles de Pesca y Acuicultura por Países. Perú. Hojas de datos de perfiles de los países. Roma. Consultado 19 julio 2018. Disponible en: http://www. fao.org/fishery/

Geat, Y.; Bauchart, D.; Hocquette, J. and Culioli, J. 2001. Effect of nutritional factors on biochemical, structural and metabolic characteristics of muscles in ruminants, consequences on dietic value and sensorial of meat. Reprod. Nutri., 41:1-26 p.

Gonçalves, A. and Duarte, J. 2009. Effects of phosphate treatment on quality of red shrimp (Pleoticus muelleri) processed with cryomechanical freezing. Food Science and tecnology., 42: 1435-1438 p.

Harries, J.; Rhodes, D. and Chrystall, B. 1972. Meat Texture. I. Subjective assessment of the texture cooked beef. Texture studies 3. 101p.

Hernández, E. 2005. Evaluación Sensorial. Facultad de Ciencias básicas e ingeniería. Bogotá Colombia. 128p.

Hevia, F.; Wilckens, R. y Badilla, R. 2001. Caracteristicas del almidon y contenido de proteína de quinua (Cheonopodium quinoa wild) cultivada bajo diferentes niveles. Agro sur. Valdivia. Chile 29:1.

Ingram, M.; Bray, D.; Clark, D.; Dolmon, C.; Elliot, R. y Thetcher, F. 1983..International comisión on Microbiological Specifications. "Microorganismos de los Alimentos. Técnicas de Análisis Microbiológicos". Vol. I. Segunda Edición. I Tittle Orginal: Microorganism in Foods. Ed. Acribia S. A. Zaragoza 106 -122p.

IMARPE. Instituto del Mar Peruano. 1996. Compendio Biológico Tecnológico de las Principales Especies Hidrobiológicas Comerciales del Perú. Perú. Editorial Stella. 146p.

Llorente, 2008. Quinoa: Un auténtico superalimento. Discovery DSalud. Disponible en: http://www.dsalud. com/index.php? pagina $=$ articulo\&c $=218$

Melgarejo, I. 2002. Elaboración de hamburguesa para consumo humano a partir de la especie hidrobiológica amazónica Prochilodus nigricans "boquichico". Trabajo de Fin de Carrera, Facultad de Ingeniería en Industrias Alimentarias, Universidad Nacional de la Amazonía Peruana (UNAP), Iquitos, Perú.

Muela et al. 2011. Muela, E., Alonso, V., Campo, M., Sanudo, C. Beltran, J. 2014. Antioxidant dict supplementation and lamb quality throughtout perservetion time. Meat Sci., 98:289 - 295.

Muñoz, A. 2011. Elaboración de conservas y cocidos cárnicos. Innovación y cualificación. Málaga, España. $22 \mathrm{p}$.

Ostolaza, 1978. Conservas de filetes de bonito en salsas no tradicionales. Universidad Nacional Agraria La Molina, Lima- Perú. Tesis para optar el título de Ingeniero Pesquero. 1-2p.

Panuncio, A.; Cardeza, L.; Quintero, M.; Solé, M.; Barrios, S. y Gámbaro, A. 2013. Efecto de la incorporación de transglutaminasa microbiana en las propiedades sensoriales de hamburguesa de minced de merluza (Merluccius hubbsi). Instituto de Investigaciones Pesqueras. Facultad de Veterinaria. UdelaR. 42p.

Pearson, D. 1993. "Técnicas de laboratorio para el análisis de alimentos" Edit. Acribia, S.A. Zaragoza. España.

Pérez, B. 2016. Tendencias en el desarrollo de productos de alimentación para el 2017. Disponible en: https:// www.ainia.es/tecnoalimentalia/

PRODUCE, 2017. Ministerio de la Producción. Anuario Estadístico Pesquero y Acuícola. Lima, Perú. 196p.

SANIPES. 2016. Manual de indicadores sanitarios y de inocuidad para los productos pesqueros y acuícolas para mercado nacional y de exportación. 70p.

Shaviklo, A. and Fahim, A. 2013. Quality improvement of silver carp fingers by optimizing the level of major elements influencing texture. Internacional Food Resarch Journal. Guilan, Iran. 283-290p.

Stejskal, V.; Vejsada, P.; Cepak, M.; špička, J.; Vacha, F.; Kouril, J. and Policar, T. 2011. Sensory and textural attributes and fatty acid profiles of fillets of extensively and intensively farmed Eurasian perch (Perca fluviatilis L.). Food Chem., 129. 1054-1059p.

Teruya, L. 1973. Procesamiento de la pasta de pescado, tipo kamaboko, de bonito (sarda chiliensis) y merluza (Merluccius gayi peruanus), sometidos a cocción por vapor. Universidad Nacional Agraria La Molina. Lima - Perú. Tesis para optar el título de Ingeniero Pesquero.

Tokur, B.; Ozkutuk, S.; Atici, E.; Ozyurt, G. and Ozyurt, C. 2006. Chemical and sensory quality change of fish fingers made from mirror capr (Cyprinus carpio), during frozen storage. Departament of fishing and fish processing technology. Universidad de Cukurova. Adana, Turquía. 335 - 341p. 